\title{
ON THE EXISTENCE OF CERTAINTY EQUIVALENTS OF VARIOUS RELEVANT TYPES
}

\author{
J. C. R. ALCANTUD AND G. BOSI
}

Received 24 July 2002 and in revised form 5 May 2003

We tackle the problem of associating certainty equivalents with preferences over stochastic situations, which arises in a number of different fields (e.g., the theory of risk attitudes or the analysis of stochastic cooperative games). We study the possibility of endowing such preferences with certainty equivalence functionals that satisfy relevant requirements (such as positive homogeneity, translation invariance, monotonicity with respect to first-order stochastic dominance, and subadditivity). Uniqueness of the functional is also addressed in fairly general conditions.

\section{Introduction}

This paper is devoted to a formal research into the problem of associating certainty equivalents with preferences over stochastic situations, thus formalizing a well-known and highly intuitive idea in decision with uncertainty that seemed to have undergone studies. Indeed, the existence of such assignements is relevant in a number of different fields, ranging from the theory of risk attitudes (cf., e.g., Fishburn [6, Section 1.6] and Kreps [7, Section 3.2]; also in a multiperiod context: cf. Epstein and Zin [5], where certainty equivalents are used as functional forms for risk preferences) to the analysis of stochastic cooperative games (cf. Suijs and Borm [14]). The common assumption of such a concept is exemplified, for example, in Luce [10], where a setting quite similar to ours (a preference over uncertain outcomes plus a certainty equivalence functional on it) is presumed as a prerequisite that permits to evaluate the joint receipt of gambles. Unlike such position, we intend to gain insight as to when such 
functional assignement is feasible in terms of the less astringent concept of preference over such uncertain situations. We here consider (a) the existence of certainty equivalence functionals, and (b) the possibility of imposing relevant properties on them, which will widen the range of applicability of that type of (nonlinear) utility assignements for the initial risky prospects.

The possibility of assigning certainty equivalents is crucial for the purpose of extending admitted models of choice behavior that depend upon the "cardinality" of the items considered for the case where uncertain alternatives (with or without knowledge or estimation of probabilities) appear. In Luce's words, what we need for the ground set is "its elements being something for which judgements of preference make sense." In accordance with the variety of situations where certainty equivalents have played important roles, several axiomatizations of this concept have been used in the literature. Thus, we will state our model and study some relationships regarding other possible variations on it. The framework will be that of an agent which has preferences on a certain collection of nonnegative real random variables. We then present a uniqueness result for certainty equivalents in fairly general conditions. Afterwards, we focus on the properties of the order under which existence is granted. Our main aim will be to obtain necessary and sufficient conditions for that unique endowment to fulfil a number of properties that are desirable in particular contexts, namely, translation invariance, positive homogeneity, monotonicity with respect to first-order stochastic dominance (cf. Whitmore and Findlay [17]), and subadditivity.

\section{Notation and preliminaries}

Throughout this paper, the following context will be assumed. We will be concerned with a fixed collection $\mathbf{K}$ of nonnegative real random variables (i.e., measurable real functions) on a given probability space $(\Omega, \mathcal{A}, \mathcal{D})$. We also assume that the collection $\overline{\mathbf{K}}$ of all the nonnegative constant random variables belongs to K. Given any real number $\lambda \geqslant 0$, the degenerate random variable equal to $\lambda$ will be denoted by $\bar{\lambda}$.

Recall that a binary relation $R$ on a set $\mathbf{A}$ is said to be a preorder if it is reflexive and transitive. For every $a \in \mathbf{A}$, define the upper contour set of $a$ as the set $\{b \in \mathbf{A}: a R b\}$. The lower contour set of any element $a \in$ $\mathbf{A}$ is defined analogously. If $\mathbf{A}$ is endowed with a topology, then $R$ is continuous whenever the upper and lower contour sets of every element $a \in \mathbf{A}$ are closed. We will study the case of a complete preorder $\preccurlyeq$ on $\mathbf{K}$. Completeness of $\preccurlyeq$ means that for any pair $X, Y \in \mathbf{K}$, either $X \preccurlyeq Y$ or $Y \preccurlyeq X$. The strict part $\prec$ and the symmetric part $\sim$ of $\preccurlyeq$ are defined in the usual way. Under our assumptions, $\sim$ is a binary equivalence relation. 
Definition 2.1. A functional $\mathcal{C}: \mathbf{K} \rightarrow \mathbb{R}$ is a certainty equivalence functional for $\preccurlyeq$ if the following conditions are met:

(i) $\mathcal{C}$ is a utility functional for $\preccurlyeq$ (i.e., for every $X, Y \in \mathbf{K}, X \preccurlyeq Y$ if and only if $\mathcal{C}(X) \leqslant \mathcal{C}(Y))$

(ii) $\mathcal{C}(\bar{\lambda})=\lambda$ for every nonnegative real number $\lambda$.

Obviously, condition (i) forces us to restrict our attention to complete preorders.

Observe that conditions (i) and (ii) above imply the following condition:

(iii) $X \sim \overline{\mathcal{C}(X)}$ for every $X \in \mathbf{K}$.

Condition (iii) means that $\mathcal{C}(X)$ is a certainty equivalent of $X$ for every $X \in \mathbf{K}$. It is the numerical level such that the agent is indifferent when receiving it with certainty and receiving a payoff determined by $X$. This amount must be necessarily unique: observe that, for each $\lambda \in \mathbb{R}_{+}$such that $X \sim \bar{\lambda}, \mathcal{C}(X)=\mathcal{C}(\bar{\lambda})$ by (i), and now (ii) entails $\mathcal{C}(X)=\lambda$.

However, there is no guarantee that (ii) holds true under (i) plus (iii), as the following example shows.

Example 2.2. Consider $\overline{\mathbf{K}}=\left\{\bar{\lambda}: \lambda \in \mathbb{R}_{+}\right\}$and the degenerate preorder $\sim$, where all the elements in $\overline{\mathbf{K}}$ are indifferent to each other. Define $\mathcal{C}(\bar{\lambda})=0$ for each $\bar{\lambda} \in \overline{\mathbf{K}}$. Now (i) and (iii) are fulfilled but (ii) is clearly false.

Our setting is a relevant (suitably modified) particular case of a more general framework which has been exposed, for example, in Luce [10, Section 2]. There, the ground set is required to include $\mathbb{R}$, while we request that a copy of a part of $\mathbb{R}$ (the nonnegative constant random variables) belongs to that set. This prompts another difference in defining what certainty equivalence means at "the numerical level": if numbers are involved, then certainty equivalents of quantities are declared as themselves (Definition 1(2) in [10], which is adapted in the form of our (ii) for constant random variables). However, these differences are basically superfluous or expository, and so virtually all the general results hold for our particular setting, perhaps in an adapted form. For example, Luce [10, Lemma 1(i)] is translated into (iii) above, and the necessary condition in Luce [10, Lemma 1(iv)] — that the binary relation must be a complete preorder-is specified as a redundant prerequisite here for the sake of clarity.

Example 2.2 shows that a utility functional that assigns the certainty equivalent to each random variable in $\mathbf{K}$ may fail to associate $\lambda$ with the sure stochastic payoff $\bar{\lambda}$. One consequence is that, in general, (i) plus (iii) do not provide a different axiomatization of our concept of certainty 
equivalence functional. In order to present natural situations where (iii) entails (ii), we will need to introduce further axioms, for example, monotonicity with respect to first-order stochastic dominance. This assumption is fairly intuitive in many relevant situations, for example, when we are concerned with risk attitudes and monetary outcomes (cf. Fishburn [6, Section 1.6]). Recall that the first-order stochastic dominance relation $\preccurlyeq_{\mathrm{FSD}}$ on $\mathbf{K}$ is the (not necessarily complete) preorder on $\mathbf{K}$ defined as follows:

$$
X \preccurlyeq \mathrm{FSD} Y \Leftrightarrow D(\{\omega \in \Omega: X(\omega) \leqslant t\}) \geqslant P(\{\omega \in \Omega: Y(\omega) \leqslant t\}) \quad \forall t \in \mathbb{R}_{+} .
$$

Whitmore and Findlay [17] display theory and applications of the concept in bulk.

A complete preorder $\preccurlyeq$ on $\mathbf{K}$ is said to be monotonic with respect to firstorder stochastic dominance if, for every $X, Y \in \mathbf{K}, X \preccurlyeq_{\mathrm{FSD}} Y$ implies $X \preccurlyeq Y$, and $X \prec_{\mathrm{FSD}} Y$ implies $X \prec Y$. Now we have the following property.

Property 2.3. Under the monotonicity of the preorder $\preccurlyeq$ with respect to first-order stochastic dominance, (iii) implies (ii).

Proof. By monotonicity of the preorder $\preccurlyeq$ with respect to first-order stochastic dominance, $\lambda<\lambda^{\prime}$ implies $\bar{\lambda}<\bar{\lambda}^{\prime}$ for $\lambda, \lambda^{\prime} \in \mathbb{R}_{+}$. By (iii), for each nonnegative real number $\lambda, \mathcal{C}(\bar{\lambda})$ is the only number satisfying $\bar{\lambda} \sim \overline{\mathcal{C}(\bar{\lambda})}$; therefore $\lambda=\mathcal{C}(\bar{\lambda})$.

However, we will not be bounded by that particular axiom along the next sections.

Although uniqueness of the certainty equivalence functional is an important issue in the current context, we have not claimed it in our definition. We proceed to show that it is implicitly required by our model.

Lemma 2.4. Let $\preccurlyeq$ be a complete preorder on any collection $\mathbf{K}$ of nonnegative real random variables on a common probability space $(\Omega, \mathcal{A}, D)$, containing all the nonnegative constants. If there exists a nonnegative certainty equivalence functional for $\preccurlyeq$, then it is unique.

Proof. By contradiction, assume that there exist two different certainty equivalence functionals $\mathcal{C}$ and $\mathcal{C}^{*}$ for $\preccurlyeq$. Then there exists $X \in \mathbf{K}$ such that $\mathcal{C}(X) \neq \mathcal{C}^{*}(X)$. Without loss of generality, assume that $\mathcal{C}(X)<\mathcal{C}^{*}(X)$, and consider any positive real number $\lambda$ with $\mathcal{C}(X)<\lambda<\mathcal{C}^{*}(X)$. Then $\mathcal{C}(X)<\mathcal{C}(\bar{\lambda})=\lambda=\mathcal{C}^{*}(\bar{\lambda})<\mathcal{C}^{*}(X)$, and this is contradictory since $\mathcal{C}$ and $\mathcal{C}^{*}$ are both utility functionals for $\preccurlyeq$. So the proof is complete. 


\section{Existence of certainty equivalents that satisfy continuity or translation invariance}

We will be concerned with the existence of different types of (unique) continuous certainty equivalence functionals in the rest of the paper. The key fact is expressed by Theorem 3.3, where we characterize the existence of a (unique) continuous certainty equivalence functional in terms of representability of the order and also of assumptions on $\preccurlyeq$. In order to achieve this latter aim, we need some definitions on preordered sets.

Definition 3.1. Let $R$ be a preorder on a set $\mathbf{A}$ and denote by $P$ its strict part. We say that $R$ is order separable (in the sense of Debreu) if there exists a countable subset $\mathbf{Z}$ of $\mathbf{A}$ such that if $a P b$, then there is $z \in \mathbf{Z}$ with $a R z R b$.

It is well known that there are alternative versions of this property (see, e.g., Bridges and Mehta [3, Proposition 1.4.4]).

Besides, as certainty equivalents are a good possibility for substituting uncertain alternatives (e.g., gambles or any other stochastic situation) by a "measure" of them in suitable models (see, e.g., Luce [10]), it is therefore relevant to know when certainty equivalents exist satisfying translation invariance (often called the axiom of monotonicity). We analyze such behavior in that same statement. By translation invariance we mean that $\mathcal{C}(X+\bar{\lambda})=\mathcal{C}(X)+\lambda$ whenever $X \in \mathbf{K}$ and $\lambda \in \mathbb{R}_{+}$, satisfying $X+\bar{\lambda} \in \mathbf{K}$. This property admits a characterization in the following terms.

LeMMA 3.2. Let $\preccurlyeq$ be a complete preorder on any collection $\mathbf{K}$ of nonnegative real random variables on a common probability space $(\Omega, \mathcal{A}, D)$, containing all the nonnegative constants.

A nonnegative certainty equivalence functional $C$ for $\preccurlyeq$ is translation invariant if and only if $X+\bar{\lambda} \sim Y+\bar{\lambda}$ for all $X \sim Y$ in $\mathbf{K}$ and $\lambda \in \mathbb{R}_{+}$with $X+\bar{\lambda}$, $Y+\bar{\lambda} \in \mathbf{K}$.

Proof. Suppose that $\mathcal{C}$ is translation invariant. If $X \sim Y$, since $\mathcal{C}$ is a utility on $\mathbf{K}$ then $\mathcal{C}(X)=\mathcal{C}(Y)$, so $\mathcal{C}(X)+\mathcal{\lambda}=\mathcal{C}(Y)+\lambda$, which now means $\mathcal{C}(X+$ $\bar{\lambda})=\mathcal{C}(Y+\bar{\lambda})$. The fact that $\mathcal{C}$ is a utility yields $X+\bar{\lambda} \sim Y+\bar{\lambda}$.

Conversely, if $X+\bar{\lambda} \sim Y+\bar{\lambda}$ for all $X \sim Y$ in $\mathbf{K}$ and $\lambda \in \mathbb{R}_{+}$with $X+\bar{\lambda}$, $Y+\bar{\lambda} \in \mathbf{K}$, then for any $X \in \mathbf{K}$ and $\lambda \in \mathbb{R}_{+}$, we deduce that $\mathcal{C}(X+\bar{\lambda})=$ $\mathcal{C}(X)+\lambda$. Indeed, now $X+\bar{\lambda} \sim \overline{\mathcal{C}(X)}+\bar{\lambda}$ (since $X \sim \overline{\mathcal{C}(X)}$ because $\mathcal{C}$ is a certainty equivalent), so these elements have the same certainty equivalent under $\mathcal{C}$. But, because $\overline{\mathcal{C}(X)}+\overline{\mathcal{\lambda}}=\overline{\mathcal{C}(X)+\lambda}$ by definition, then $\mathcal{C}(X+$ $\bar{\lambda})=\mathcal{C}(X)+\lambda$ using (ii) in the definition of certainty equivalent.

THEOREM 3.3. Let $\preccurlyeq$ be a complete preorder on any collection $\mathbf{K}$ of nonnegative real random variables on a common probability space $(\Omega, \mathcal{A}, D)$, containing all 
the nonnegative constants, and let $\tau_{\mathbf{K}}$ be any topology on $\mathbf{K}$ for which scalar multiplication is continuous (e.g., a vector topology). Assume that $\overline{0} \preccurlyeq X$ for every $X \in \mathbf{K}$. Then the following conditions are equivalent:

(i) there exists a unique nonnegative continuous (resp., translation invariant) certainty equivalence functional $\mathcal{C}$ for $\preccurlyeq$;

(ii) the following conditions are verified:

(a) $\preccurlyeq$ is representable by a continuous utility functional $C^{\prime}$,

(b) $\lambda_{1}<\lambda_{2} \Rightarrow \bar{\lambda}_{1} \prec \bar{\lambda}_{2}$ for all $\lambda_{1}, \lambda_{2} \in \mathbb{R}_{+}$,

(c) for every $X \in \mathbf{K}$, there exists $\lambda \in \mathbb{R}_{+}$such that $X \sim \bar{\lambda}$ (resp., (c') $X+\bar{\lambda} \sim Y+\bar{\lambda}$ for all $X \sim Y$ in $\mathbf{K}$ and $\lambda \in \mathbb{R}_{+}$with $X+\bar{\lambda}, Y+\bar{\lambda} \in$ $\mathbf{K})$;

(iii) the following conditions are verified:

(d) $\preccurlyeq$ is separable and continuous,

(e) $\lambda_{1}<\lambda_{2} \Rightarrow \bar{\lambda}_{1} \prec \bar{\lambda}_{2}$ for every $\lambda_{1}, \lambda_{2} \in \mathbb{R}_{+}$,

(f) for every $X \in \mathbf{K}$, there exists $\lambda \in \mathbb{R}_{+}$such that $X \sim \bar{\lambda}$ (resp., $\left(\mathrm{f}^{\prime}\right)$ $X+\bar{\lambda} \sim Y+\bar{\lambda}$ for all $X \sim Y$ in $\mathbf{K}$ and $\lambda \in \mathbb{R}_{+}$with $X+\bar{\lambda}, Y+\bar{\lambda} \in$ K).

Proof. It is clear that (i) implies (ii). Observe that $X \sim \overline{\mathcal{C}(X)}$ for every $X \in \mathbf{K}$, by (iii) following the definition of certainty equivalent, which yields (c). If the additional property $\mathcal{C}(X+\bar{\lambda})=\mathcal{C}(X)+\lambda$ for all $X \in \mathbf{K}$ and $\lambda \in \mathbb{R}_{+}$holds, then ( $\left.c^{\prime}\right)$ must also hold by Lemma 3.2.

Now, we show that (ii) implies (i). Uniqueness holds by Lemma 2.4. Furthermore, by our assumptions, there exists a nonnegative continuous utility functional $\mathcal{C}^{\prime}$ for $\preccurlyeq$. Without loss of generality, we can assume that $\mathcal{C}^{\prime}(\overline{0})=0$ so that $\mathcal{C}^{\prime}$ is nonnegative. Define the bijection $I: \overline{\mathbf{K}} \rightarrow[0, \infty[$ by

$$
I(\bar{\lambda})=\lambda \quad\left(\lambda \in \mathbb{R}_{+}\right),
$$

which is continuous by the continuity of scalar multiplication under $\tau_{\mathbf{K}}$. Further, define the function $\phi: \mathbb{R}_{+} \rightarrow \mathbb{R}_{+}$by

$$
\phi:=\mathcal{C}_{\mid \overline{\mathrm{K}}}^{\prime} \circ I^{-1}
$$

Observe that, by condition (b), $\phi$ is strictly increasing since $\mathcal{C}^{\prime}$ is a utility functional for $\preccurlyeq$. Then $\phi^{-1}$ exists and it is strictly increasing and continuous. Indeed, $\phi^{-1}$ is the inverse of a strictly increasing continuous realvalued function defined on $\mathbb{R}_{+}$. We claim that the functional $\mathcal{C}=\phi^{-1} \circ \mathcal{C}^{\prime}$ on $\mathbf{K}$ is a nonnegative continuous certainty equivalence functional for $\preccurlyeq$. First observe that $\mathcal{C}$ is well defined since the image set $\phi\left(\mathbb{R}_{+}\right)$(the domain of $\left.\phi^{-1}\right)$ is equal to the image set $\mathcal{C}^{\prime}(\mathbf{K})$ by condition (c). It is clear that $\mathcal{C}$ is nonnegative and continuous (recall that $\mathcal{C}^{\prime}$ is continuous). Further, $\mathcal{C}$ is a utility functional for $\preccurlyeq$ since it is a strictly increasing transformation 
of the utility functional $\mathcal{C}^{\prime}$. Finally, for every $\lambda \in \mathbb{R}_{+}$, it is

$$
\mathcal{C}(\bar{\lambda})=I \circ \mathcal{C}_{\left.\right|_{\bar{K}}}^{\prime-1} \circ \mathcal{C}^{\prime}(\bar{\lambda})=I(\bar{\lambda})=\lambda .
$$

If, additionally, ( $\left.c^{\prime}\right)$ holds, for any $X \in \mathbf{K}$ and $\lambda \in \mathbb{R}_{+}$, we deduce that $\mathcal{C}(X+\bar{\lambda})=\mathcal{C}(X)+\lambda$ using Lemma 3.2.

The equivalence of (ii) and (iii) is well known. We address the reader to Bridges and Mehta [3, Theorem 3.2.9] in order to check that (a) is equivalent to $(\mathrm{d})$.

Because we are only concerned with continuous certainty equivalence functionals, we have not made explicit the result parallel to Theorem 3.3, where "continuous" is dropped elsewhere. Nonetheless, that development is analogous to ours. If the continuity assumption is ommited, the topology on $\mathbf{K}$ becomes irrelevant.

Remark 3.4. Theorem 3.3(b) holds when the complete preorder $\preccurlyeq$ on $\mathbf{K}$ is monotonic with respect to first-order stochastic dominance. Indeed, when this happens, we have that

$$
\lambda_{1}<\lambda_{2} \Longrightarrow \bar{\lambda}_{1} \prec_{\mathrm{FSD}} \bar{\lambda}_{2} \Longrightarrow \bar{\lambda}_{1}<\bar{\lambda}_{2} \quad \forall \lambda_{1}, \lambda_{2} \in \mathbb{R}_{+} .
$$

In the following example, we show that there exists a continuously representable complete preorder satisfying Theorem 3.3(b) which does not admit a (continuous) certainty equivalence functional.

Example 3.5. Let $\mathbf{K}$ be the real convex cone of nonnegative real random variables on a common probability space $(\Omega, \mathcal{A}, D)$ which is generated by a nonnegative and nondegenerate real random variable $Z$ and the constant $\overline{1}$. Therefore, a random variable $X$ belongs to $K$ if and only if there exist two nonnegative real numbers $\lambda$ and $\mu$ such that $X=\lambda Z+\bar{\mu}$. Consider the positive real-valued function $U$ on $\mathbf{K}$ defined by $U(X)=$ $U(\lambda Z+\bar{\mu})=\lambda+1-e^{-\mu}$ and let $\preccurlyeq$ be the complete preorder on $\mathbf{K}$ defined by

$$
X \preccurlyeq Y \Longleftrightarrow U(X) \leqslant U(Y)
$$

Then $\preccurlyeq$ satisfies Theorem 3.3(a) and (b). Nevertheless, a nondegenerate random variable $\lambda Z$ does not admit a certainty equivalent if $\lambda$ is greater than one. Indeed, if $\lambda$ is a real number greater than one, then 
$\lambda Z$ is strictly preferred to every constant since we have that $U(\lambda Z)=\lambda>$ $1-e^{-\mu}=U(\bar{\mu})$ for every constant $\bar{\mu}$.

\section{Existence of a continuous and positively homogeneous certainty equivalent}

In this section, we consider positively homogeneous certainty equivalence functionals: these are functionals $\mathcal{C}: \mathbf{K} \rightarrow \mathbb{R}_{+}$for which $\mathcal{C}(\lambda X)=\lambda \mathcal{C}(X)$ for every positive real number $\lambda$. When dealing with risk aversion, some authors refer to as constant relative risk aversion instead (see, e.g., Epstein and Zin [5]). Now we are able to apply the representability result of Bosi et al. [2] in order to characterize the existence of a continuous certainty equivalence functional that also satisfies this property. We will provide a solution in terms of the following homotheticity condition as applied to the preference over stochastic situations.

Definition 4.1. A preorder $R$ on a real cone $\mathbf{A}$ in a real vector space $\mathbf{V}$ is said to be homothetic if, for every $x, y \in \mathbf{A}$ and $\lambda \in \mathbb{R}_{++}, x R y$ is equivalent to $\lambda x R \lambda y$.

It is noted that homotheticity keeps being an important issue in other different realms, including both production (cf. Varian [16], Silva and Stefanou [12]) and demand analysis (cf. Varian [15], Liu and Wong [9]), as well as macroeconomics (cf. Lewbel [8]) or general equilibrium models (cf. Préchac [11]).

Proposition 4.2. Let $\preccurlyeq$ be a complete preorder on a real cone $\mathbf{K}$ in a topological vector space $\mathbf{X}$ of nonnegative real random variables on a common probability space $(\Omega, \mathcal{A}, D)$. Assume that $\mathbf{K}$ contains all the nonnegative constants, it is endowed with the induced topology $\tau_{\mathbf{K}}$, and $\overline{0} \preccurlyeq X$ for every $X \in \mathbf{K}$. Then the following assertions are equivalent:

(i) there exists a unique nonnegative, positively homogeneous, and continuous certainty equivalence functional $\mathcal{C}$ for $\preccurlyeq$;

(ii) there exists a nonnegative, positively homogeneous, and continuous utility functional $\mathcal{C}^{\prime}$ for $\preccurlyeq$, and $\mathcal{C}^{\prime}(\overline{1}) \neq 0$;

(iii) the following conditions are verified:
(a) $\preccurlyeq$ is homothetic,
(b) $\preccurlyeq$ is continuous,
(c) $\overline{0} \prec \overline{1}$.

Proof. It is clear that (i) implies (ii) and (ii) implies (iii). We prove that (iii) implies (i). By conditions (a) and (b), we have that the complete preorder $\preccurlyeq$ is representable by a nonnegative, positively homogeneous, and 
continuous utility functional $\mathcal{C}^{\prime}$ (see the corollary in Bosi et al. [2]). By condition $(c)$, we have that $\mathcal{C}^{\prime}(\overline{1}) \neq 0$. Define a real-valued functional $\mathcal{C}$ on $\mathbf{K}$ by $\mathcal{C}(\cdot)=\left(1 / \mathcal{C}^{\prime}(\overline{1})\right) \mathcal{C}^{\prime}(\cdot)$. It is immediate to check that $\mathcal{C}$ is a nonnegative, positively homogeneous, and continuous certainty equivalence functional for $\preccurlyeq$. Obviously, uniqueness is guaranteed by Lemma 2.4.

Remark 4.3. We stress that condition (c) is essential in the previous proposition. Should we drop it, the statement becomes false, as the degenerate preorder $\preccurlyeq$ defined by $X \sim Y$ for every $X, Y \in \mathbf{K}$ proves. This preorder has a unique positively homogeneous utility functional $\mathcal{C}^{\prime} \equiv 0$ which happens to be continuous and nonnegative, but it does not admit a certainty equivalence functional because of condition (b) in Theorem 3.3.

Remark 4.4. The separability condition that is needed in Theorem 3.3 is obviously implicit in the requirements of Proposition 4.2. That is, under Proposition 4.2(iii), the order must also be separable. Similar issues were already made apparent in Bosi [1] and Bosi et al. [2].

\section{Existence of certainty equivalents that are monotonic with respect to first-order stochastic dominance}

We now consider another property of certainty equivalence functionals that is desirable in some interesting frameworks: monotonicity with respect to first-order stochastic dominance. For example, in decision theory under uncertainty, this property arises quite naturally in the most classical linear utility model: the expected utility theory of von Neumann and Morgenstern. A functional $\mathcal{C}: \mathbf{K} \rightarrow \mathbb{R}_{+}$is said to be monotonic with respect to first-order stochastic dominance if, for every $X, Y \in \mathbf{K}, X \preccurlyeq{ }_{F S D} Y$ implies $\mathcal{C}(X) \leqslant \mathcal{C}(Y)$, and $X \prec_{\text {FSD }} Y$ implies $\mathcal{C}(X)<\mathcal{C}(Y)$.

Corollary 5.1. Let $\preccurlyeq$ be a complete preorder on a real cone $\mathbf{K}$ in a topological vector space $\mathbf{X}$ of nonnegative real random variables on a common probability space $(\Omega, \mathcal{A}, D)$. Assume that $\mathbf{K}$ contains all the nonnegative constants and endow it with the induced topology $\tau_{\mathrm{K}}$. Then the following assertions are equivalent:

(i) there exists a unique nonnegative, positively homogeneous, and continuous certainty equivalence functional $\mathcal{C}$ for $\preccurlyeq$ that is monotonic with respect to first-order stochastic dominance;

(ii) there exists a unique nonnegative, positively homogeneous, and continuous utility functional $\mathcal{C}^{\prime}$ for $\preccurlyeq$ that is monotonic with respect to first-order stochastic dominance, and $\mathcal{C}^{\prime}(\overline{1}) \neq 0$;

(iii) the following conditions are verified: 
(a) $\preccurlyeq$ is homothetic,

(b) $\preccurlyeq$ is continuous,

(c) $\preccurlyeq$ is monotonic with respect to first-order stochastic dominance.

Proof. Observe that first-order stochastic dominance monotonicity of the preorder $\preccurlyeq$ implies the condition $\overline{0} \preccurlyeq X$ for every $X \in \mathbf{K}$, as well as $\overline{0} \prec \overline{1}$. The thesis now follows immediately from Proposition 4.2.

\section{A characterization of subadditive certainty equivalents}

Our study will end with the analysis of subadditivity. We recall that a real-valued functional $\mathcal{C}$ on a real convex cone $\mathbf{A}$ in a real vector space $\mathbf{V}$ is said to be subadditive if $\mathcal{C}(x+y) \leqslant \mathcal{C}(x)+\mathcal{C}(y)$ for all $x, y \in \mathbf{A}$. Then the following proposition characterizes certainty equivalence functionals which are subadditive.

Proposition 6.1. Let $\preccurlyeq$ be a complete preorder on a real convex cone $\mathbf{K}$ in a topological vector space $\mathbf{X}$ of nonnegative real random variables on a common probability space $(\Omega, \mathcal{A}, \mathcal{D})$. Assume that $\mathbf{K}$ contains all the nonnegative constants, and $\overline{0} \preccurlyeq X$ for every $X \in \mathbf{K}$. If there exists a nonnegative certainty equivalence functional $\mathcal{C}$ for $\preccurlyeq$, then $\mathcal{C}$ is subadditive if and only the following property holds for all $X, Y \in \mathbf{K}$ and $\lambda, \mu \in \mathbb{R}_{++}$:

(i) $X \preccurlyeq \bar{\lambda}, Y \preccurlyeq \bar{\mu}$ imply $X+Y \preccurlyeq \overline{\lambda+\mu}$.

Proof. Let $\mathcal{C}$ be a subadditive certainty equivalence functional for $\preccurlyeq$. Then for every $X, Y \in \mathbf{K}$ and $\lambda, \mu \in \mathbb{R}_{++}$such that $X \preccurlyeq \bar{\lambda}$ and $Y \preccurlyeq \bar{\mu}$, we have that $\mathcal{C}(X+Y) \leqslant \mathcal{C}(X)+\mathcal{C}(Y) \leqslant \mathcal{\lambda}+\mu=\mathcal{C}(\overline{\lambda+\mu})$, which implies $X+$ $Y \preccurlyeq \overline{\lambda+\mu}$. Hence, condition (i) holds. Conversely, assume that condition (i) holds and that $\mathcal{C}$ is a nonnegative certainty equivalence functional for $\preccurlyeq$. We show that $C$ must be also subadditive. By contradiction, assume that there exist $X, Y \in \mathbf{K}$ such that $\mathcal{C}(X)+\mathcal{C}(Y)<\mathcal{C}(X+Y)$. Since $\mathcal{C}$ is nonnegative, we may consider two positive real numbers $\lambda, \mu$ such that $\mathcal{C}(X)<\lambda, \mathcal{C}(Y)<\mu$, and $\lambda+\mu<\mathcal{C}(X+Y)$. Then we have that $X<\bar{\lambda}$ and $Y<\bar{\mu}$, but $\overline{\lambda+\mu}<X+Y$, and this contradicts condition (i) above. This consideration ends the proof.

The concept of a subadditive certainty equivalence functional is closely linked to the concept of a risk loving complete preorder. This is a complete preorder $\preccurlyeq$ on a collection $\mathbf{K}$ of nonnegative real random variables with finite expectations on a probability space $(\Omega, \mathcal{A}, D)$ such that $\overline{\mathbb{E}(X)} \preccurlyeq X$ for all $X \in \mathbf{K}$, where $\mathbb{E}(\cdot)$ is the expectation operator on $\mathbf{K}$. In this case any stochastic payoff $X$ is weakly preferred to its expectation (see, e.g., Suijs [13]). 
We conclude this paper by presenting an example of a continuous, positively homogeneous, translation invariant, and subadditive certainty equivalence functional for a risk loving complete preorder.

Example 6.2. Let $\preccurlyeq$ be a complete preorder on a real convex cone $\mathbf{K}$ of nonnegative real random variables on a common probability space $(\Omega, \mathcal{A}, D)$ containing all the nonnegative constants. Assume that there exists a concave probability distortion $g$ (i.e., a nondecreasing, concave, and continuous function $g:[0,1] \rightarrow[0,1]$ such that $g(0)=0$ and $g(1)=1$ ) such that the Choquet integral

$$
\int_{\Omega} X d g \circ D=\int_{0}^{\infty} g \circ D(\{X \geq t\}) d t
$$

is finite for all $X \in \mathbf{K}$ and the real-valued functional $\mathcal{C}$ on $\mathbf{K}$ defined by $\mathcal{C}(X)=\int_{\Omega} X d g \circ D$ for all $X \in \mathbf{K}$ is a utility functional for $\preccurlyeq$. Then $\mathcal{C}$ is a positively homogeneous, translation invariant, and subadditive certainty equivalence functional for the complete preorder $\preccurlyeq$ (see, e.g., Denneberg [4]), which is risk loving since $g(p) \geq p$ for all $p \in[0,1]$. Further, $\mathcal{C}$ is continuous with respect to the norm topology corresponding to the norm $\|X\|=\int_{\Omega} X d g \circ D$ on $\mathbf{K}$ (see again Denneberg [4, Proposition 9.4]).

\section{Acknowledgments}

The work of J. C. R. Alcantud has been supported by the Junta de Castilla y León under the Research Project SA061/02 and by FEDER and DGICYT Project PB98-551. Most of this research was done while this author was visiting the Dipartimento di Matematica Applicata "Bruno de Finetti," to whom he is indebted for their hospitality and support. The authors gratefully acknowledge comments from an anonymous referee that contributed to improve the original manuscript with substantial additions.

\section{References}

[1] G. Bosi, A note on the existence of continuous representations of homothetic preferences on a topological vector space, Ann. Oper. Res. 80 (1998), 263-268.

[2] G. Bosi, J. C. Candeal, and E. Induráin, Continuous representability of homothetic preferences by means of homogeneous utility functions, J. Math. Econom. 33 (2000), no. 3, 291-298.

[3] D. S. Bridges and G. B. Mehta, Representations of Preferences Orderings, Lecture Notes in Economics and Mathematical Systems, vol. 422, Springer-Verlag, Berlin, 1995. 


\section{Continuous certainty equivalence}

[4] D. Denneberg, Non-Additive Measure and Integral, Theory and Decision Library. Series B: Mathematical and Statistical Methods, vol. 27, Kluwer Academic Publishers, Dordrecht, 1994.

[5] L. G. Epstein and S. E. Zin, "First-order" risk aversion and the equity premium puzzle, J. Monetary Econ. 26 (1990), no. 3, 387-407.

[6] P. C. Fishburn, Nonlinear Preference and Utility Theory, Johns Hopkins Series in the Mathematical Sciences, vol. 5, Johns Hopkins University Press, Maryland, 1988.

[7] D. M. Kreps, A Course in Microeconomic Theory, Harvester Wheatsheaf, New York, 1990.

[8] A. Lewbel, Aggregation without separability: a generalized composite commodity theorem, Amer. Econ. Rev. 86 (1996), no. 3, 524-543.

[9] P.-W. Liu and K.-C. Wong, Revealed homothetic preference and technology, J. Math. Econom. 34 (2000), no. 3, 287-314.

[10] R. D. Luce, Joint receipt and certainty equivalents of gambles, J. Math. Psych. 39 (1995), no. 1, 73-81.

[11] C. Préchac, Monotonicity of equilibrium prices with respect to aggregate resources, Econom. Lett. 60 (1998), no. 1, 63-66.

[12] E. Silva and S. E. Stefanou, Generalization of nonparametric tests for homothetic production, Amer. J. Agr. Econ. 78 (1996), 542-546.

[13] J. Suijs, Cooperative Decision-Making under Risk, Kluwer Academic Publishers, Dordrecht, 2000.

[14] J. Suijs and P. Borm, Stochastic cooperative games: superadditivity, convexity, and certainty equivalents, Games Econom. Behav. 27 (1999), no. 2, 331-345.

[15] H. R. Varian, The nonparametric approach to demand analysis, Econometrica 50 (1982), no. 4, 945-973.

[16] , The nonparametric approach to production analysis, Econometrica 52 (1984), no. 3, 579-597.

[17] G. A. Whitmore and M. C. Findlay (eds.), Stochastic Dominance: An Approach to Decision Making under Risk, D. C. Heath and Company, Massachusetts, 1978.

J. C. R. Alcantud: Facultad de Economía y Empresa, Universidad de Salamanca, E 37008 Salamanca, Spain

E-mail address: jcr@usal.es

G. Bosi: Dipartimento di Matematica Applicata "Bruno de Finetti," Università di Trieste, Piazzale Europa 1, 34127 Trieste, Italy

E-mail address: giannibo@econ.univ.trieste.it 


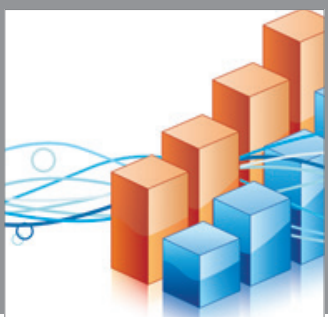

Advances in

Operations Research

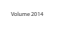

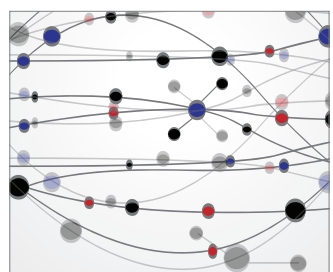

\section{The Scientific} World Journal
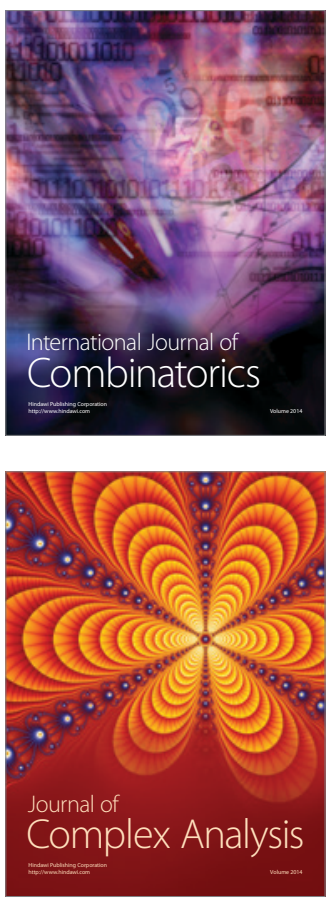

International Journal of

Mathematics and

Mathematical

Sciences
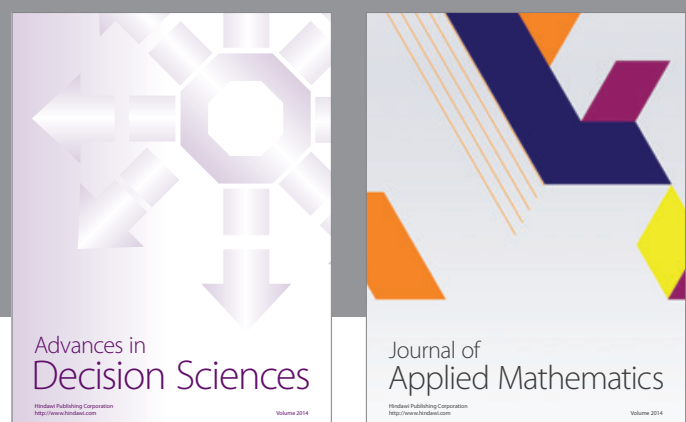

Journal of

Applied Mathematics
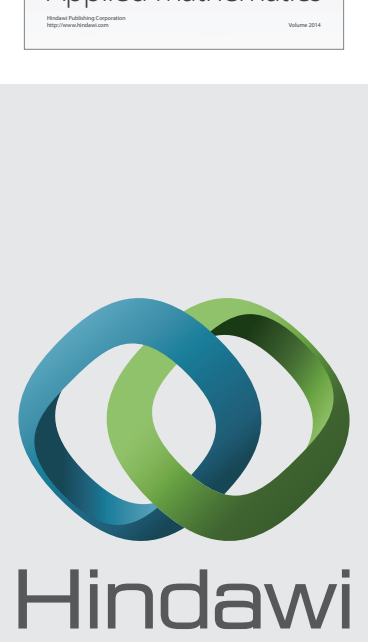

Submit your manuscripts at http://www.hindawi.com
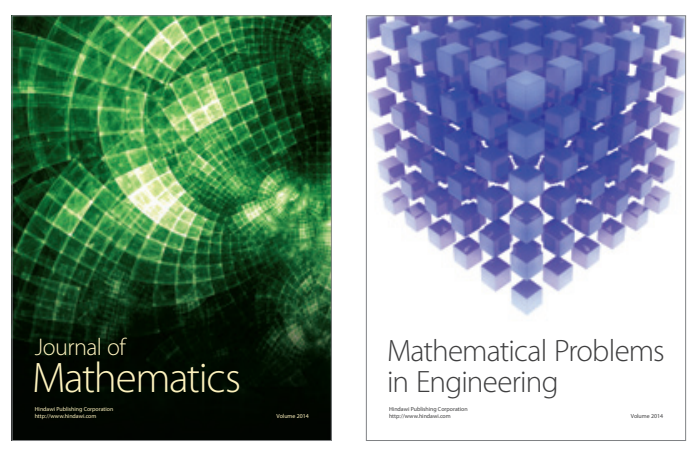

Mathematical Problems in Engineering
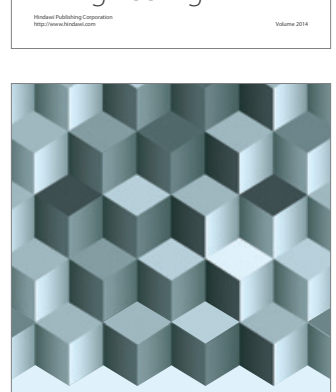

Journal of

Function Spaces
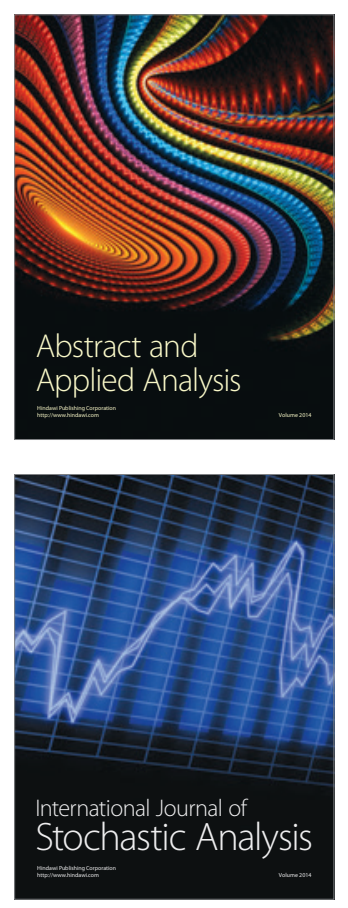

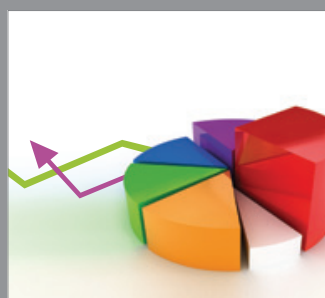

ournal of

Probability and Statistics

Promensencen
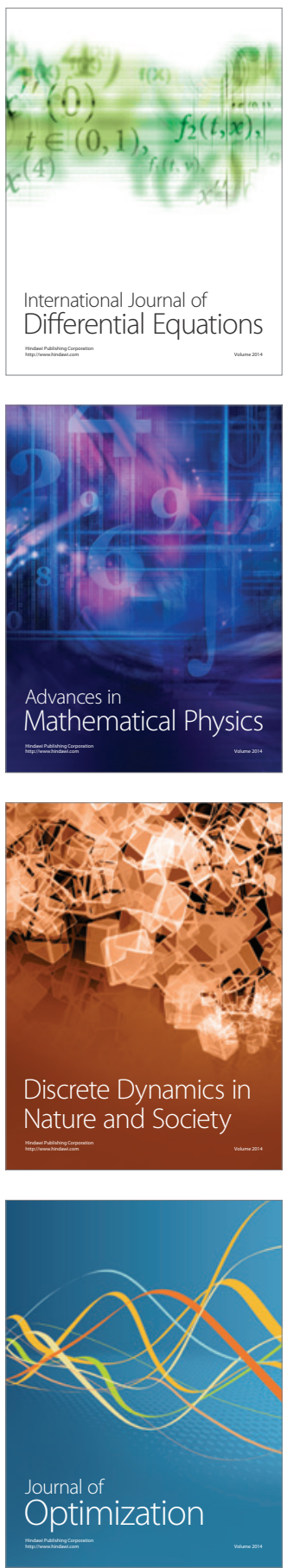